\title{
Frontières
}

\section{HARRISON, Robert, Les morts, traduit de l'américain par Florence Naugrette avec la collaboration de Guillaume Maurice, Paris, Le Pommier, 2003, 288 p.}

\section{Jean-Jacques Lavoie}

Volume 16, numéro 2, printemps 2004

URI : https://id.erudit.org/iderudit/1074127ar

DOI : https://doi.org/10.7202/1074127ar

Aller au sommaire du numéro

Éditeur(s)

Université du Québec à Montréal

ISSN

1180-3479 (imprimé)

1916-0976 (numérique)

Découvrir la revue

Citer ce compte rendu

Lavoie, J.-J. (2004). Compte rendu de [HARRISON, Robert, Les morts, traduit de l'américain par Florence Naugrette avec la collaboration de Guillaume Maurice, Paris, Le Pommier, 2003, 288 p.] Frontières, 16(2), 99-100. https://doi.org/10.7202/1074127ar d'utilisation que vous pouvez consulter en ligne.

https://apropos.erudit.org/fr/usagers/politique-dutilisation/ 


\section{HARRISON, Robert}

\section{Les morts}

traduit de l'américain par Florence Naugrette avec la collaboration de Guillaume Maurice Paris, Le Pommier, 2003, 288 p.

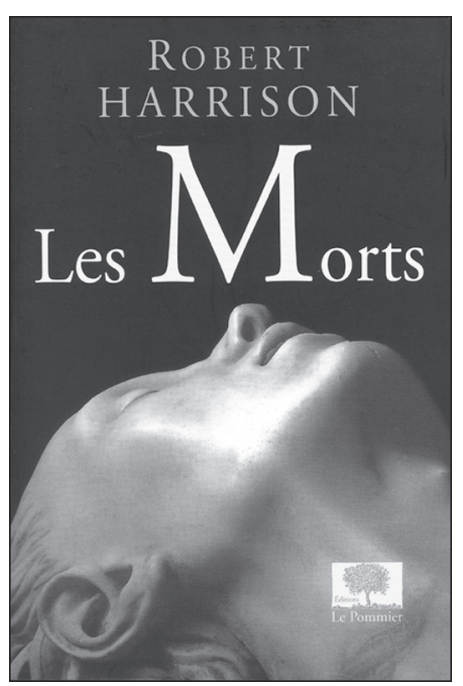

Notre civilisation occidentale a produit maints exemples de pratiques visant à permettre aux vivants de maintenir une relation avec leurs morts. Il existerait des lieux, des topoi, où les morts exercent leur pouvoir, formulent leurs exigences, prennent la parole et, plus généralement, cohabitent avec les vivants. L'ouvrage de Robert Harrison, directeur du département de littérature française et italienne de I'Université de Stanford (Californie), s'intéresse à quelquesuns de ces endroits où les morts mènent leur vie posthume séculière: les tombes, les maisons, les lois, les mots, les images, les rêves, les rituels, etc. Plus précisément, l'auteur s'intéresse à la nature et au fonctionnement de ces divers habitats des morts dans le monde des vivants.

Bien que plusieurs thèses se croisent dans cet essai, on peut dire que la thèse principale est la suivante: les vivants n'enterrent pas les morts simplement pour les maintenir à l'écart par une séparation étanche, mais aussi et surtout pour humaniser le sol sur lequel ils construisent leur monde et fondent leur histoire. Être humain, c'est donc avant toute chose enterrer ses morts et, par conséquent, la sépulture peut être considérée, selon l'auteur, comme l'institution originelle de la nature humaine.

Pour bien souligner ce lien intime entre les humains et l'humus, le premier thème abordé est celui de la terre comme corollaire élémentaire de l'Histoire. Contrairement à la mer qui n'offre aucun appui à l'existence du monde humain et qui représente l'agent imaginaire de l'oubli ultime, la terre, solide et stable, autorise I'inscription qui fonde l'histoire et la mémoire. Seule la terre peut recevoir une pierre tombale, car la mer efface tout, refuse toute écriture et rend impossible le repérage de l'endroit où la disparition a eu lieu; elle est comme une tombe infinie qui reste vierge de toute marque humaine.

Le second thème abordé est celui de la puissance fondatrice de la tombe. À la suite de Heidegger, Harrison est d'avis que si l'être humain est un être-au-monde, alors rien n'est aussi intimement relié à notre être que nos pratiques de construction, car le monde où nous sommes jetés est toujours un monde bâti. Toutefois, contrairement à Heidegger qui déclarait que «seul un dieu peut nous sauver ", Harrison conclut plutôt que c'est l'architecte qui peut sauver l'être humain du déracinement heideggerien, car seule l'architecture a en son pouvoir d'héberger notre mortalité.

Ce premier désaccord avec Heidegger - et ce ne sera pas le seul - n'empêche pas Harrison de poursuivre sa réflexion sur les traces de ce « philosophe de la mort le plus radical de la pensée occidentale " (p. 137). Il est bien connu que ce dernier a affirmé que « le langage est la maison de l'être » et que cette affirmation devait être comprise au pied de la lettre: c'est donc en déterminant ce qu'est l'essence d'une maison que l'on peut arriver à savoir ce qu'est l'être. C'est pourquoi le nouveau thème qui préoccupe Harrison prend la forme d'une question qui peut paraître insolite: "Qu'est-ce qu'une maison? » La réponse qu'il donne à cette question se fonde sur un rappel historique : à l'origine, les êtres humains ont d'abord construit des maisons pour leurs morts avant de s'en construire pour eux-mêmes. Puis, pour les Grecs et les Romains, loin d'être d'abord un abri, la maison fut essentiellement un lieu de cohabitation entre les vivants et les morts. Enfin, l'auteur résume luimême sa thèse dans ces quelques mots: " une maison est un lieu d'intériorité, au sein de la nature, où les morts, grâce aux soins des vivants, continuent à vivre après leur mort et défendent les intérêts de ceux qui sont encore à naître » (p. 63).

Harrison se propose ensuite de montrer que notre relation à la mort passe d'abord par notre relation aux morts, selon des modes que Heidegger n'envisagea jamais ou qu'il ignorait tout simplement. Selon Harrison, Heidegger commet une erreur sérieuse dans Être et Temps quand il affirme que le cadavre est une simple chose, qui dans sa présence là-devant ne donne au Dasein (le là de l'être ou l'être-là ou encore l'être dans-le-monde) aucun accès à sa propre mort. Au contraire, malgré son immobilité pesante, Harrison montre bien que rien n'est plus dynamique qu'un cadavre. C'est l'événement du passage qui se déroule sous nos yeux. Voici ce qu'il dit à ce sujet: "Ce phénomène du passage [...] fait du corps sans vie une " chose " relationnelle qui, dans sa sujétion au pouvoir de la mort, lie ensemble le passé, le présent et l'avenir. Le passé (le ne-plus-être-ici de la personne), le présent (le cadavre dans son être-là-devant) et l'avenir (le destin qui attend ceux qui marchent sur les pas du défunt) convergent tous dans le corps mort, au moins tant qu'il reste l'objet de I'attention et de la sollicitude des vivants » (p. 141-142).

Harrison est donc également en désaccord avec Heidegger lorsque ce dernier affirme que le Dasein ne peut pas approcher sa propre mort par la mort des autres. Pour Harrison, affirmer que la mort est non relationnelle est tout aussi faux que de réduire le cadavre à un simple être-là-devant. Sur ce point, il préfère suivre la thèse de Freud et de Jung selon laquelle l'être humain ne possède pas la notion innée de sa propre mort ou, pour le dire autrement, que l'inconscient n'a aucune idée de sa propre mortalité puisqu'il se croit immortel. II en résulte donc que seule la mort de l'être aimé - institutionnalisée et ritualisée dans les funérailles, la sépulture, les lamentations... - nous persuade contre nos convictions les plus instinctives que nous sommes des êtres-pour-la-mort.

Qui plus est, Harrison est d'avis que le Dasein continue à habiter le cadavre jusqu'à ce que le lien soit coupé par ceux qui ont survécu. La vérité de cette proposition, à savoir que «le Dasein ne meurt pas avant 
qu'on ait disposé de ses restes " (p. 213), repose sur une certitude: l'obligation envers le cadavre ou envers ses restes se retrouve dans l'ensemble des cultures humaines. C'est d'ailleurs pourquoi, depuis toujours, il est si difficile de faire le travail de deuil en l'absence du corps. C'est ce qu'illustraient déjà très bien les Tragiques grecs et c'est aussi ce que ne cesse de confirmer l'état psychique fragile des proches de soldats disparus au combat, de victimes d'accidents d'avion ou des camps de la mort.

Bien entendu, Harrison aborde aussi certains thèmes qui n'ont rien de heideggerien. C'est le cas, par exemple, du lien caché entre le chagrin et la vocalisation humaine, et de l'importance de la lamentation ritualisée. Une fois de plus, ces deux thèmes donnent lieu à deux thèses. En ce qui concerne le premier thème, Harrison est d'avis que c'est probablement le chagrin qui a offert à la voix humaine ses premiers accents dans les registres du tragique. Quant à la thèse reliée au second thème, elle est plus facilement vérifiable: "les lamentations rituelles sont des gestes d'externalisation [sic] appris et hautement formalisés, dont le but est avant tout de dépersonnaliser le chagrin en soumettant ce dernier à un ensemble de codes transmis par la tradition. [...] La ritualisation sert ainsi à contenir la crise du chagrin via l'acte même d'objectiver son contenu par des gestes convenus et des codes précis » (p. 88). Les anthropologues (par exemple, RadcliffBrown, Langer, Lévi-Strauss, Bloch, Moore et bien d'autres) ont en effet bien mis en évidence que l'objectivation a une double fonction: d'une part, elle empêche le chagrin de dégénérer en émotivité chaotique et non maîtrisée et, d'autre part, elle offre à la communauté la possibilité de partager la plainte des affligés.

Les anthropologues, les écrivains, les philosophes et les historiens ne sont pas les seuls à être investis d'une autorité particulière dans le cadre des réflexions touffues de ce livre. Certains exégètes du Nouveau Testament sont aussi convoqués afin d'élucider la contradiction apparente entre une théologie de la tombe vide de Jésus, qui, selon Harrison, est au fondement de la révolution chrétienne, et le culte chrétien des corps saints et des reliques. Enfin, certains éléments des héritages chrétiens et préchrétiens sont aussi sollicités pour mieux comprendre le monument le plus visité de l'Amérique : le Mémorial des vétérans du Viêtnam, qui, avec sa liste de 59000 noms dont l'âge moyen est de dix-neuf ans, réussit à particulariser le général et dans le même temps à refléter le général dans le particulier.

En définitive, bien que l'édition française ait supprimé une grande partie des notes de l'édition anglaise, on ne peut que remercier les responsables des éditions Le Pommier d'avoir rendu accessibles au public francophone ces méditations qui nous éclairent, par le biais de petits et ravissants voyages dans notre patrimoine littéraire, philosophique et religieux, sur la façon dont nous partageons notre monde avec ceux qui nous ont précédés.

Jean-Jacques Lavoie 